Pacific Journal of Mathematics

SUMS OF PRODUCTS OF POWERS OF GIVEN PRIME

ROB TIJDMANAND LION KIANG WANG 


\section{SUMS OF PRODUCTS OF POWERS OF GIVEN PRIME NUMBERS}

\section{R. TiJdeman AND LianXiang WANG}

We give the complete solutions of the equations $2^{x} 3^{y}+1=2^{z}+$ $3^{w}, 2^{x} 3^{y}+2^{z}=3^{w}+1$ and $2^{x} 3^{y}+3^{w}=2^{z}+1$ in integers $x, y, z, w$. We use this to prove that every large rational number has at most four representations of the form $2^{\alpha} 3^{\beta}+2^{\gamma}+3^{\delta}$. Finally we prove that, for given integer $n$ and prime numbers $p_{1}, \ldots, p_{t}$, every rational number $m$ has at most $C$ representations of the form $\sum_{i=1}^{n} p_{1}^{k_{11}} \cdots p_{t}^{k_{i t}}$ where $k_{i 1}, \ldots, k_{i t}$ are integers.

0. D. J. Newman conjectured that if $w(n)$ denotes the number of solutions of $n=2^{a}+3^{b}+2^{c} 3^{d}$ then $w(n)$ is bounded (see Erdös and Graham [4] p. 80). Evertse, Györy, Stewart and Tijdeman [6] Theorem 6(a) settled this conjecture. We call two representations $x_{1}+\cdots+x_{n}$ and $x_{1}^{\prime}+\cdots+x_{n}^{\prime}$ distinct, if the unordered tuples $\left(x_{1}, \ldots, x_{n}\right)$ and $\left(x_{1}^{\prime}, \ldots, x_{n}^{\prime}\right)$ are not the same. In $\S 2$ we prove that the number of distinct representations of a rational number $m$ as $2^{\alpha} 3^{\beta}+2^{\gamma}+3^{\delta}$ is at most four, if $m$ exceeds a certain constant. The number four is the best possible.

To prove this result we need not only the Main Theorem on $S$-Unit Equations (Lemma 4) as in [6], but also the complete solutions of the diophantine equations mentioned in the first paragraph of this paper. Here we recall the remark of Brenner and Foster ([2] Comment 8.037) that the class of equations

$$
1+(p q)^{a}=p^{b}+q^{c}
$$

where $p, q$ are given distinct primes, does not seem to be amenable by their (congruential) method. We show in $\S 1$ how the more general equation

$$
1+p^{x} q^{y}=p^{z}+q^{w}
$$

can be treated by Baker's method for estimating linear forms in the logarithms of algebraic numbers. The essential tool in $\S 1$ is Lemma 1, due to Ellison [3] and specially made for the primes $p=2, q=3$. De Weger [10] has proved a corresponding result for all primes $p, q$ with $2 \leq p<q \leq 200$ and the method works for any pair of prime 
numbers. Thus the methods in $\S \S 1$ and 2 extend to any pair of prime numbers $p, q$ in place of 2,3 . The referee kindly pointed out to the authors that the methods used in $\S 1$ are quite similar to the following result, which is Theorem 2.3 in Paul Vojta's thesis [8]. In particular, Vojta gives an effective procedure for obtaining our Theorems 1-3, although he does not actually carry out this procedure.

TheOREM (Vojta [8]). Let $S$ be a finite set of places of $\mathbf{Z}$ containing at most 3 elements. Fix integers $a, b, c, d$. Then there are only finitely many solutions to the equation $a x+b y+c z+d=0$ in $S$-units $x, y, z$; and these solutions can be effectively bounded in terms of $a, b, c, d$, and $S$.

The crucial point is that the set $S$ can contain at most 3 elements.

In a letter to one of us, $P$. Erdós asked whether we can prove that every integer has at most $C$ representations of the form

$$
2^{\alpha} 3^{\beta} 5^{\gamma}+2^{\delta} 3^{\varepsilon}+2^{\zeta} 5^{\theta}+\cdots+3^{\lambda}+5^{\mu} .
$$

We prove in $\S 3$ the much more general result that for any positive integer $n$ and any prime numbers $p_{1}, \ldots, p_{t}$ there exists a number $C$ such that every rational number $m$ has at most $C$ representations of the form $\sum_{i=1}^{n} p_{1}^{k_{i 1}} \ldots p_{t}^{k_{t t}}$ where $k_{i 1}, \ldots, k_{i t}$ are integers. Some weaker results in this direction are given in $[6, \S 6]$. We cannot answer Erdós' question in a later letter to find a number $C$ which depends only on $n$ and $t$ (hence is independent of the primes $p_{1}, \ldots, p_{t}$ ), since the corresponding problem for the Main Theorem on $S$-Unit Equations has not been solved yet. In Theorem 6 we extend the above mentioned results to algebraic number fields.

1. The diophantine equations $2^{x} 3^{y}+1=2^{z}+3^{w}, 2^{x} 3^{y}+2^{z}=$ $3^{w}+1$ and $2^{x} 3^{y}+3^{w}=2^{z}+1$. Let $\mathbf{Z}$ denote the set of rational integers, and $\mathbf{N}_{0}$ the set of non-negative rational integers. We call the solutions $(x, y, z, w)=(x, 0, x, 0)$ and $(0, y, 0, y)$ for the first equation, $=(0, y, 0, y)$ for the second equation and $=(x, 0, x, 0)$ for the third equation (where $x, y \in \mathbf{Z}$ ) the trivial solutions. We shall determine all non-trivial solutions in $\mathbf{Z}^{4}$.

\section{THEOREM 1. The equation}

$$
2^{x} 3^{y}+1=2^{z}+3^{w}
$$


has exactly twelve non-trivial solutions $(x, y, z, w) \in \mathbf{Z}^{4}$ :

$$
\begin{aligned}
& (1,1,2,1),(1,2,4,1),(2,0,1,1),(2,1,2,2),(3,1,4,2),(3,2,6,2), \\
& (4,0,3,2),(4,2,6,4),(5,1,4,4),(-2,2,-2,1),(2,-1,1,-1), \\
& (6,-2,3,-2) .
\end{aligned}
$$

\section{TheOrem 2. The equation}

$$
2^{x} 3^{y}+2^{z}=3^{w}+1
$$

has exactly eight non-trivial solutions $(x, y, z, w) \in \mathbf{Z}^{4}$ :

$$
\begin{aligned}
& (1,0,1,1),(1,0,3,2),(1,1,2,2),(1,2,6,4), \\
& (2,1,4,3),(3,0,1,2),(3,1,2,3),(-1,1,-1,0) .
\end{aligned}
$$

THEOREM 3. The equation

$$
2^{x} 3^{y}+3^{w}=2^{z}+1
$$

has exactly nine non-trivial solutions $(x, y, z, w) \in \mathbf{Z}^{4}$ :

$$
\begin{aligned}
& (1,0,2,1),(1,1,3,1),(1,1,5,3),(1,5,9,3),(3,0,4,2) \\
& (3,1,5,2),(4,1,7,4),(4,3,9,4),(3,-1,1,-1) .
\end{aligned}
$$

The following lemma, proved by Baker's method for estimating linear forms in logarithms of algebraic numbers, is basic for the proofs.

LEMMA 1. If $x, y \in \mathbf{N}_{0}$ with $x>10$, then

$$
\left|2^{x}-3^{y}\right|>\exp (x(\log 2-0.1))
$$

apart from the exceptional pairs $(x, y)=(10,6),(11,7),(13,8),(14,9)$, $(16,10),(19,12),(27,17)$.

Proof. For $x>27$ this was proved by Ellison [3] (cf. de Weger [10], Theorem 4.3). It is easy to check the remaining range $10 \leq x \leq 27$.

The next result is due to L. Hebreus who lived around 1300 .

LEMMA 2. The equation $2^{x}+1=3^{y}$ has exactly two solutions $(x, y) \in \mathbf{Z}^{2}$, namely $(1,1)$ and $(3,2)$.

The equation $3^{y}+1=2^{x}$ has exactly two solutions $(x, y) \in \mathbf{Z}^{2}$, namely $(1,0)$ and $(2,1)$.

Proof. See Alex [1] Lemma 2.1. 
Proof of Theorem 1. First we find all solutions in $\mathbf{N}_{0}^{4}$. It is obvious from (1.1) that $x=0$ if and only if $z=0$, and that $w=0$ implies $y=0$. In these cases we have trivial solutions only. Further, if $z=1$ in $(1.1)$, then $y=0$, hence, by Lemma $2,(x, y, z, w)=(2,0,1,1)$ is the only non-trivial solution. From now on we assume that $x \geq 1, y \geq 0$, $z \geq 2, w \geq 1$.

From $(1.1)$ and $\min (y, w) \geq 1$, we see that

$$
3^{\min (y, w)} \mid 2^{z}-1
$$

which implies

$$
2 \cdot 3^{\min (y, w)-1} \leq z
$$

Hence

$$
\min (y, w)<\log z+0.4 \text {. }
$$

Similarly, from (1.1) and $\min (x, z) \geq 2$,

$$
2^{\min (x, z)} \mid 3^{w}-1
$$

which implies

$$
2^{\min (x, z)-2} \leq w
$$

Therefore

$$
\min (x, z)<(\log 2)^{-1} \log w+2 .
$$

We distinguish between four cases.

Case 1. $y<\log z+0.4$ and $x<(\log 2)^{-1} \log w+2$.

If $z \leq w$, then (1.1) implies

$$
3^{w-\log z-0.4}<3^{w-y}<2^{x}<2^{(\log 2)^{-1} \log w+2},
$$

hence

$$
w<2 \log w+1.7
$$

We infer

$$
2 \leq z \leq w \leq 4
$$

If $z>w$, then (1.1) implies

$$
2^{z-(\log 2)^{-1} \log w-2}<2^{z-x}<3^{y}<3^{\log z+0.4},
$$

hence

$$
z<3.1 \log z+2.7
$$

We infer

$$
1 \leq w<z \leq 9
$$


By (1.4) and (1.5) we find in Case 1:

$$
\begin{cases}y<\log 9+0.4, & \text { hence } 0 \leq y \leq 2, \\ x<\log 8 / \log 2+2, & \text { hence } 1 \leq x \leq 4 .\end{cases}
$$

By (1.4), (1.5) and (1.6) all solutions of (1.1) in Case 1 are contained in the list: $(x, y, z, w)=(1,1,2,1),(1,2,4,1),(2,1,2,2),(3,1,4,2)$, $(3,2,6,2),(4,0,3,2),(4,2,6,4)$.

Case 2. $y<\log z+0.4$ and $z<(\log 2)^{-1} \log w+2$.

Clearly, if $w=1$, then $z=1, y=0, x=2$. Thus we may assume $w \geq 2$. We have

$$
\begin{aligned}
y & <\log z+0.4<\log \left((\log 2)^{-1} \log w+2\right)+0.4 \\
& <\log \log w+2<0.1 w+2 .
\end{aligned}
$$

Since (1.1) implies $3^{w-y}<2^{x}$, we obtain

$$
0.9 w-2<w-y<(\log 2 / \log 3) x,
$$

hence

$$
w<0.8 x+2.3
$$

If $x>9$, then we have by Lemma 1 for non-exceptional pairs $(x, w-y)$ :

$$
\exp (x(\log 2-0.1))<\left|2^{x}-3^{w-y}\right| \leq 2^{z}-1<2^{(\log 2)^{-1} \log w+2}
$$

and so, by (1.7)

$$
x(\log 2-0.1)<\log w+1.4<\log (0.8 x+2.3)+1.4 .
$$

This implies $x \leq 5$ which is a contradiction. For the exceptional pairs we find $10 \leq x \leq 27,2 \leq w \leq 23,2 \leq z \leq 6,0 \leq y \leq 2$. The exceptional pairs $(x, w)=(10,6+y),(11,7+y),(13,8+y),(14,9+$ $y),(16,10+y),(19,12+y),(27,17+y)$ with $0 \leq y \leq 2$ do not yield new solutions of $(1,1)$. If $1 \leq x \leq 9$, then $2 \leq w \leq 9$ by (1.7), hence $2 \leq z \leq 5,0 \leq y \leq 2$. In these ranges we find the solutions $(x, y, z, w)=(2,1,2,2),(3,1,4,2),(4,0,3,2),(5,1,4,4)$.

Case 3. $w<\log z+0.4$ and $x<(\log 2)^{-1} \log w+2$.

In this case we have

$$
x<1.5 \log \log z+3 .
$$

If $z-x>27$, then it follows from (1.1) and (1.8) and Lemma 1 that

$$
\begin{aligned}
& \exp ((z-1.5 \log \log z-3)(\log 2-0.1)) \\
& \quad<\left|2^{z-x}-3^{y}\right| \leq 3^{w} / 2<3^{\log z+0.4} / 2 .
\end{aligned}
$$


Hence

$$
z<1.9 \log x+1.5 \log \log z+3.4
$$

and therefore $z \leq 8$, which yields a contradiction.

If $z-x \leq 27$, then $2 \leq z \leq 31$ by (1.8), hence $1 \leq w \leq 3,1 \leq x \leq 3$. By (1.1) we have

$$
y \leq \log \left(2^{31}+3^{3}\right) / \log 3<19.6 .
$$

Thus $0 \leq y \leq 19$. By checking the ranges for $x, y, z, w$ we find that the solutions of (1.1) in Case 3 are contained in the list: $(x, y, z, w)=$ $(1,1,2,1),(1,2,4,1),(2,1,2,2),(3,1,4,2),(3,2,6,2)$.

Case 4. $w<\log z+0.4$ and $z<(\log 2)^{-1} \log w+2$.

In this case we have

$$
z<(\log 2)^{-1}(\log (\log z+0.4))+2<(\log 2)^{-1} \log \log z+3 .
$$

Hence $2 \leq z \leq 3, w=1$. By (1.1), $2^{x} 3^{y}<2^{3}+3^{1}=11$, hence $1 \leq x \leq 3,0 \leq y \leq 2$. There is only one solution in these ranges, $(x, y, z, w)=(1,1,2,1)$. We conclude that equation (1.1) has exactly nine non-trivial solutions $(x, y, z, w) \in \mathbf{N}_{0}^{4}$, namely

$$
\begin{aligned}
& (1,1,2,1),(1,2,4,1),(2,0,1,1),(2,1,2,2),(3,1,4,2), \\
& (3,2,6,2),(4,0,3,2),(4,2,6,4),(5,1,4,4) .
\end{aligned}
$$

Next, we consider the case in which some of $x, y, z, w$ are negative. It is easy to verify that there are only trivial solutions if $x<0$ and $y \leq 0$ and if $x \leq 0$ and $y<0$. Hence we may assume $x y<0$.

Case (i). $x<0$ and $y>0$.

We have

$$
3^{y}+2^{|x|}=2^{|x|+z}+2^{|x|} 3^{w} .
$$

Thus $w>0$ and $|x|+z=0$. Therefore $x=z$. It follows from (1.9) that the equation

$$
2^{|x|} 3^{w}+1=2^{|x|}+3^{y}
$$

has only one solution $(|x|, w,|x|, y)=(2,1,2,2)$. Hence $(-2,2,-2,1)$ is the only non-trivial solution of (1.1) in Case (i).

Case (ii). $x>0$ and $y<0$.

In this case we have

$$
2^{x}+3^{|y|}=2^{z} 3^{|y|}+3^{|y|+w} .
$$


Then $z>0$ and $|y|+w=0$, hence $w=y$. From (1.9) we see that the equation

$$
2^{z} 3^{|y|}+1=2^{x}+3^{|y|}
$$

has exactly two solutions $(z,|y|, x,|y|)=(1,1,2,1),(3,2,6,2)$. Therefore $(2,-1,1,-1)$ and $(6,-2,3,-2)$ are the only non-trivial solutions of (1.1) in case (ii).

The proofs of Theorems 2 and 3 are somewhat simpler than the proof of Theorem 1, since we can use the following lemma.

LEMMA 3. (a) If $2^{a} \mid 3^{b}+1$, then $a \leq 2$. (b) If $3^{b} \mid 2^{a}+1$, then $b \leq 3$.

Proof. (a) The assertion is true for $b=0$ and $b=1$, and $3^{b+2} \equiv 3^{b}$ $(\bmod 8)$. (b) The assertion is true for $a=0,1, \ldots, 53$ and $2^{a+54} \equiv 2^{a}$ $(\bmod 81)$.

Proof of Theorem 2. First we consider all solutions in $\mathbf{N}_{0}^{4}$. We find only trivial solutions if $z=0$ or $w=0$. Thus we may assume that $x \geq 0, y \geq 0, z \geq 1, w \geq 1$. By (1.2) and Lemma 3(a),

$$
\min (x, z) \leq 2 \text {. }
$$

By (1.2) and $\min (y, w) \geq 1$, we derive from $3^{\min (y, w)} \mid 2^{z}-1$ that $2 \cdot 3^{\min (y, w)-1} \leq z$, hence

$$
\min (y, w)<\log z+0.4
$$

We again distinguish four cases.

Case $1.0 \leq x \leq 2$ and $0 \leq y<\log z+0.4$.

If $z>9$ and $(z, w)$ is a non-exceptional pair, then, by Lemma 1,

$$
\exp (z(\log 2-0.1))<\left|2^{z}-3^{w}\right|<2^{x} 3^{y}<2^{2} 3^{\log z+0.4},
$$

hence

$$
z<2 \log z+3.1 \text {. }
$$

This implies $z \leq 6$ which yields a contradiction. For each exceptional pair $(z, w)$ the number $3^{w}-2^{z}+1$ has a prime factor greater than 3 , so that there are no solutions of (1.2) in Case 1 with $z>9$. If $1 \leq z \leq 9$, then $0 \leq y \leq 2$. By (1.2) we have $3^{w}<2^{2} 3^{2}+2^{9}$, hence $1 \leq w \leq 5$. By simple computations we find that the solutions in Case 2 are contained in the list:

$$
(x, y, z, w)=(1,0,1,1),(1,0,3,2),(1,1,2,2),(1,2,6,4),(2,1,4,3) .
$$


Case 2. $0 \leq x \leq 2$ and $w<\log z+0.4$. By (1.2) we have

$$
2^{z}<3^{w}<3^{\log z+0.4}
$$

and so $z=1$. Hence $w=0$. This is impossible.

Case 3. $1 \leq z \leq 2$ and $y<\log z+0.4$.

If $z=1$, then $y=0$ and, by Lemma 2, we find the two solutions $(x, y, z, w)=(1,0,1,1),(3,0,1,2)$.

If $z=2$, then $0 \leq y \leq 1$. If $y=0$, then $3^{w}-2^{x}=3$ which is impossible. If $y=1$, then $3^{w}-3 \cdot 2^{x}=3$, hence $3^{w-1} \mid 2^{x}+1$. By Lemma $3(\mathrm{~b})$ we have $1 \leq w \leq 4$. Since $2^{x}<3^{w-1}$, we find $x \leq 4$. Now we obtain the following solutions of $(1.2):(x, y, z, w)=$ $(1,1,2,2),(3,1,2,3)$.

Case 4. $1 \leq z \leq 2$ and $w<\log z+0.4$.

Thus $w=1$. By (1.2) we have $3^{y}<3^{w}$. Hence $y=0$. This yields the solution $(1,0,1,1)$.

We conclude that (1.2) has exactly seven non-trivial solutions $(x, y, z, w) \in \mathbf{N}_{0}^{4}$, namely

$$
\begin{aligned}
& (1,0,1,1),(1,0,3,2),(1,1,2,2),(1,2,6,4) \\
& (2,1,4,3),(3,0,1,2),(3,1,2,3) .
\end{aligned}
$$

The argument for solutions with some negative values is similar to that in the proof of Theorem 1. Using (1.10) we obtain only one additional non-trivial solution in $\mathbf{Z}^{4}$, namely $(-1,1,-1,0)$.

Proof of Theorem 3. Without loss of generality we may assume that $x \geq 1, y \geq 0, z \geq 2, w \geq 1$. By (1.3) and Lemma 3(b), we have

$$
\min (y, w) \leq 3 .
$$

By (1.3) and $\min (x, z) \geq 2$, we derive from $2^{\min (x, z)} \mid 3^{w}-1$, that $2^{\min (x, z)-2} \leq w$, hence

$$
\min (x, z)<(\log 2)^{-1} \log w+2 .
$$

We again distinguish between four cases:

Case $1.0 \leq y \leq 3$ and $1 \leq x<(\log 2)^{-1} \log w+2$.

Since (1.3) implies $3^{w}<2^{z}$, we have $w<0.631 z$ and

$$
\left|2^{z}-3^{w}\right|<2^{x} 3^{y}<2^{(\log 2)^{-1} \log w+2} 3^{3}=108 w<69 z .
$$


If $z>11$, then, from (1.11) and Lemma 1, we obtain for non-exceptional pairs $(z, w)$,

$$
z(\log 2-0.1)<\log \left|2^{z}-3^{w}\right|<\log z+4.3 \text {. }
$$

Hence $z \leq 11$, which yields a contradiction. For each exceptional pair $(z, w)$ the number $2^{z}-3^{w}+1$ has some prime factor greater than 3 . Thus there are no solutions in this case with $z>11$.

If $2 \leq z \leq 11$, then $0 \leq w<0.631 z<6.95$, hence $1 \leq x \leq 4$. By checking these ranges for $x, y, z, w$ we find the solutions: $(1,0,2,1)$, $(1,1,3,1),(1,1,5,3),(3,0,4,2),(3,1,5,2),(4,1,7,4),(4,3,9,4)$.

Case 2. $0 \leq y \leq 3$ and $2 \leq z<(\log 2)^{-1} \log w+2$.

By (1.3), we have

$$
3^{w}<2^{z}<2^{(\log 2)^{-1} \log w+2}
$$

hence $w=1$ and therefore $z=1$, which is impossible.

Case 3. $1 \leq w \leq 3$ and $1 \leq x<(\log 2)^{-1} \log w+2$.

Hence $1 \leq x \leq 3$. Obviously $z \geq x$. It follows from (1.3) that

$$
\left|2^{z-x}-3^{y}\right| \leq\left|3^{w}-1\right| / 2 \leq 13 \text {. }
$$

If $z-x>9$, then we obtain from Lemma 1 for non-exceptional pairs $(z-x, y)$ that $(z-3)(\log 2-0.1) \leq \log 13$. This implies $z \leq 7$ which yields a contradiction. It is easy to check that $\left|2^{z-x}-3^{y}\right| \geq 13$ for each exceptional pair $(z-x, y)$. Thus each solution of (1.3) in Case 3 satisfies $z-x \leq 9$, hence $z \leq 12$. If $2 \leq z \leq 12$, then, by (1.3), $0 \leq y \leq 7$. We find that all solutions in this case are contained in the list: $(1,0,2,1),(1,1,3,1),(1,1,5,3),(1,5,9,3),(3,0,4,2),(3,1,5,2)$.

Case 4. $1 \leq w \leq 3$ and $2 \leq z<(\log 2)^{-1} \log w+2$.

Hence $1 \leq z \leq 3,1 \leq x \leq 3,0 \leq y \leq 1$. These ranges are covered in Case 1.

We conclude that (1.3) has exactly eight non-trivial solutions $(x, y, z, w) \in \mathbf{N}_{0}^{4}$, namely

$$
\begin{aligned}
& (1,0,2,1),(1,1,3,1),(1,1,5,3),(1,5,9,3) \\
& (3,0,4,2),(3,1,5,2),(4,1,7,4),(4,3,9,4) .
\end{aligned}
$$

The argument for solutions with some negative values is similar to that in the proof of Theorem 1. Using (1.12) we obtain only one additional non-trivial solution in $Z^{4}$, namely $(3,-1,1,-1)$.

2. The number of representations of the form $2^{\alpha} 3^{\beta}+2^{\gamma}+3^{\delta}$. In this section we tacitly assume that the numbers $\alpha, \beta, \gamma, \delta$ in a representation $2^{\alpha} 3^{\beta}+2^{\gamma}+3^{\delta}$ are integers. 
THEOREM 4. There exists a real number $M$ such that every rational number $m>M$ that admits more than three distinct representations $2^{\alpha} 3^{\beta}+2^{\gamma}+3^{\delta}$ is of the form $2^{a}+3^{b}$. If $m=2^{a}+3^{b}$, then the representations are given by

$$
\begin{aligned}
2^{a-1} 3^{0}+2^{a-1}+3^{b} & =2^{a-2} 3^{1}+2^{a-2}+3^{b}=2^{1} 3^{b-1}+2^{a}+3^{b-1} \\
& =2^{3} 3^{b-2}+2^{a}+3^{b-2}
\end{aligned}
$$

The proof of Theorem 4 is based on the following version of the Main Theorem on $S$-Unit Equations:

LEMMA 4. Let $p_{1}, \ldots, p_{t}$ be prime numbers. There are only finitely many rational numbers $x_{0}, x_{1}, \ldots, x_{n}$ each of the form $\pm p_{1}^{k_{1}} \cdots p_{t}^{k_{t}}$ $\left(k_{1}, \ldots, k_{t} \in \mathbf{Z}\right)$ such that

$$
\begin{gathered}
\min _{0 \leq j \leq n}\left(\left|\operatorname{ord}_{p_{t}}\left(x_{j}\right)\right|\right)=0, \quad(i=1, \ldots, t), \\
x_{0}+x_{1}+\cdots+x_{n}=0,
\end{gathered}
$$

but

$$
\begin{array}{ll}
x_{i_{1}}+\cdots+x_{i_{k}} \neq 0 \quad \text { for each proper, non-empty subset } \\
\\
\left\{i_{1}, \ldots, i_{k}\right\} \text { of }\{0,1, \ldots, n\} .
\end{array}
$$

Proof. See van der Poorten and Schlickewei [7] and Evertse [5]. We express (2.3) succinctly by saying that no subsum of $x_{0}+\cdots+x_{n}$ vanishes.

We shall further use the following lemma, the proof of which is based on Lemma 1 like the proofs of Theorems 1-3.

LEMMA 5. The equation $2^{x}+3^{y}=2^{z}+3^{w}$ has exactly five solutions in integers with $x>z, y<w$, namely

$$
(x, y, z, w)=(2,0,1,1),(3,1,1,2),(4,0,3,2),(5,1,3,3),(8,1,4,5) .
$$

Proof. The case $y>0, z>0$ has been treated by Stroeker and Tijdeman [9], §10, Ex. 1. There are no solutions with $y<0$ or $z<0$. If $y=0$ or $z=0$, then $(x, y, z, w)$ satisfies $2^{x} 3^{y}+1=2^{z}+3^{w}$ or $2^{x}+3^{y}=1+2^{z} 3^{w}$ and the conclusions follow from Theorem 1 .

Proof of Theorem 4. Suppose $m$ has two distinct representations

$$
m=2^{\alpha_{1}} 3^{\beta_{1}}+2^{\gamma_{1}}+3^{\delta_{1}}=2^{\alpha_{2}} 3^{\beta_{2}}+2^{\gamma_{2}}+3^{\delta_{2}} .
$$


By applying Lemma 4 to

$$
2^{\alpha_{1}} 3^{\beta_{1}}+2^{\gamma_{1}}+3^{\delta_{1}}-2^{\alpha_{2}} 3^{\beta_{2}}-2^{\gamma_{2}}-3^{\delta_{2}}
$$

we find that $m \leq M_{1}$ for some absolute constant $M_{1}$ if (2.5) has no vanishing subsums. Let $m>M_{1}$. Since the representations are distinct, $m$ has exactly two vanishing subsums, which are complementary. Either both vanishing subsums have three terms or one subsum has two and the other four.

(a) Both vanishing subsums have three terms. After interchanging the subscripts, if necessary, we are in one of the following cases:

(a1) $2^{\alpha_{1}} 3^{\beta_{1}}+2^{\gamma_{1}}-2^{\alpha_{2}} 3^{\beta_{2}}=0,3^{\delta_{1}}-2^{\gamma_{2}}-3^{\delta_{2}}=0$,

(a2) $2^{\alpha_{1}} 3^{\beta_{1}}+2^{\gamma_{1}}-2^{\gamma_{2}}=0,3^{\delta_{1}}-2^{\alpha_{2}} 3^{\beta_{2}}-3^{\delta_{2}}=0$,

(a3) $2^{\alpha_{1}} 3^{\beta_{1}}+2^{\gamma_{1}}-3^{\delta_{2}}=0,3^{\delta_{1}}-2^{\alpha_{2}} 3^{\beta_{2}}-2^{\gamma_{2}}=0$,

(a4) $2^{\alpha_{1}} 3^{\beta_{1}}+3^{\delta_{1}}-2^{\alpha_{2}} 3^{\beta_{2}}=0,2^{\gamma_{1}}-2^{\gamma_{2}}-3^{\delta_{2}}=0$,

(a5) $2^{\alpha_{1}} 3^{\beta_{1}}+3^{\delta_{1}}-2^{\gamma_{2}}=0,2^{\gamma_{1}}-2^{\alpha_{2}} 3^{\beta_{2}}-3^{\delta_{2}}=0$,

(a6) $2^{\gamma_{1}}+3^{\delta_{1}}-2^{\alpha_{2}} 3^{\beta_{2}}=0,2^{\alpha_{1}} 3^{\beta_{1}}-2^{\gamma_{2}}-3^{\delta_{2}}=0$.

By applying Lemma 4 in Cases (a3), (a5) and (a6), we see that $\alpha_{1}, \beta_{1}, \gamma_{1}, \delta_{1}, \alpha_{2}, \beta_{2}, \gamma_{2}, \delta_{2}$ are all bounded, whence $m$ is bounded by $M_{2}$ say. We assume $m>M_{2}$. We treat the other cases separately.

Case (a1). We have $3^{\delta_{1}}=2^{\gamma_{2}}+3^{\delta_{2}}$. Hence $\delta_{1}>0, \delta_{2}=0$. By Lemma 2 , we obtain $\left(\delta_{1}, \gamma_{2}, \delta_{2}\right)=(1,1,0)$ or $(2,3,0)$. Furthermore $2^{\alpha_{1}} 3^{\beta_{1}}+2^{\gamma_{1}}=2^{\alpha_{2}} 3^{\beta_{2}}$. Let $A=\min \left(\alpha_{1}, \gamma_{1}, \alpha_{2}\right)$ (may be negative). At least two among $\alpha_{1}, \gamma_{1}, \alpha_{2}$ are equal to $A$. If $\alpha_{2}=A$ then $\beta_{2}>0$, hence $\beta_{1}=0$. Thus we have $2^{\gamma_{1}-A}+1=3^{\beta_{2}}$ or $2^{\alpha_{1}-A}+1=3^{\beta_{2}}$ and, by Lemma $2, \beta_{2} \in\{1,2\}$. Otherwise $\alpha_{1}=\gamma_{1}=A$. Then $3^{\beta_{1}}+1=2^{\alpha_{2}-A} 3^{\beta_{2}}$, hence $\beta_{1} \in\{0,1\}, \beta_{2}=0$, or $\beta_{1}=\beta_{2}=-1$. We conclude that in Case (a1) $m$ can be written as $2^{a} 3^{b}+c$ with $a \in \mathbf{Z}, b \in\{-1,0,1,2\}, c \in\{3,9\}$.

Case (a4). We have $2^{\gamma_{1}}=2^{\gamma_{2}}+3^{\delta_{2}}$, hence, by Lemma $2,\left(\gamma_{1}, \gamma_{2}, \delta_{2}\right)=$ $(1,0,0)$ or $(2,0,1)$. Further $2^{\alpha_{1}} 3^{\beta_{1}}+3^{\delta_{1}}=2^{\alpha_{2}} 3^{\beta_{2}}$. By a similar reasoning as in Case (a1) we find that in Case (a4) the number $m$ can be written as $2^{a} 3^{b}+c$ with $a \in\{-3,-1,0,1,2\}, b \in \mathbf{Z}, c \in\{2,4\}$. Z.

Case (a2). We conclude that $m$ can be written as $2^{a}+3^{b}$ with $a, b \in$

(b) One vanishing subsum has two and the other has four terms. After interchanging the subscripts and rewriting terms, if necessary, we are in one of the following cases:

(b1) $2^{\alpha_{1}} 3^{\beta_{1}}=2^{\alpha_{2}} 3^{\beta_{2}}, 2^{\gamma_{1}}+3^{\delta_{1}}=2^{\gamma_{2}}+3^{\delta_{2}}, \alpha_{1} \neq 0, \beta_{1} \neq 0$,

(b2) $2^{\gamma_{1}}=2^{\gamma_{2}}, 2^{\alpha_{1}} 3^{\beta_{1}}+3^{\delta_{1}}=2^{\alpha_{2}} 3^{\beta_{2}}+3^{\delta_{2}}$, 
(b3) $3^{\delta_{1}}=3^{\delta_{2}}, 2^{\alpha_{1}} 3^{\beta_{1}}+2^{\gamma_{1}}=2^{\alpha_{2}} 3^{\beta_{2}}+2^{\gamma_{2}}$.

Case (b1). We have $\alpha_{1}=\alpha_{2}, \beta_{1}=\beta_{2}$. Since we started with distinct representations, we may further assume $\gamma_{1}>\gamma_{2}, \delta_{1}<\delta_{2}$. By Lemma 5 ,

$$
\begin{aligned}
\left(\gamma_{1}, \delta_{1}, \gamma_{2}, \delta_{2}\right)= & (2,0,1,1),(3,1,1,2),(4,0,3,2),(5,1,3,3), \\
& \text { or }(8,1,4,5) .
\end{aligned}
$$

Thus $m$ is of the form $2^{a} 3^{b}+c$ with $a, b \in \mathbf{Z}, c \in\{5,11,17,35,259\}$.

Case (b2). We have $\gamma_{1}=\gamma_{2}$. Since the representations are distinct, we may assume $\delta_{1}<\delta_{2}$. Put $B=\min \left(\beta_{1}, \delta_{1}, \beta_{2}\right)$. Then $2^{\alpha_{1}} 3^{\beta_{1}-B}+$ $3^{\delta_{1}-B}=2^{\alpha_{2}} 3^{\beta_{2}-B}+3^{\delta_{2}-B}$. Note that at least two among $\beta_{1}, \delta_{1}, \beta_{2}$ equal $B$. If $\beta_{1}=\delta_{1}=B$, then Theorem 3 implies $2^{\alpha_{1}} 3^{\beta_{1}-B}+3^{\delta_{1}-B} \in$ $\{3,5,9,17,33,129,513\}$. If $\beta_{1}=\beta_{2}=B$, then Lemma 5 implies $2^{\alpha_{1}} 3^{\beta_{1}-B}+3^{\delta_{1}-B} \in\{5,11,17,35,259\}$. If $\delta_{1}=\beta_{2}=B$, then Theorem 1 implies $2^{\alpha_{1}} 3^{\beta_{1}-B}+3^{\delta_{1}-B} \in\{7 / 3,13 / 4,5,7,73 / 9,13,17,19,25,73,97$, 145\}. Factors 3 can be combined with $3^{B}$. We conclude that in Case (b2) $m$ is of the form $2^{a}+3^{b} c$ with $a, b \in \mathbf{Z}, c \in\{1,13 / 4,5,7,11,13$, $17,19,25,35,43,73,97,145,259\}$.

Case (b3). We have $\delta_{1}=\delta_{2}$. By a similar reasoning as in Case (b2), but with applying Theorem 2 in place of Theorem 3, we can show that $m$ is of the form $2^{a} c+3^{b}$ with $a, b \in \mathbf{Z}, c \in\{1,7 / 3,5,7,73 / 9,11,13$, $17,19,25,35,41,73,97,145,259\}$.

In each case we have found a representation of $m$ as sum of two terms, e.g. $2^{a}+3^{b} c$ in Case (b2). We call the constructed representation a common pairing of the representation (2.4). The common pairing is obtained from each of these representations by taking two terms together. Note that in each case the common pairing consists of two terms each of which has only prime factors less than 300 and such that $\min _{1 \leq j \leq 2}\left(\left|\operatorname{ord}_{p}\left(x_{j}\right)\right|\right)$ is bounded. It now follows from Lemma 4 that for $m$ large, $m>M_{3}$ say, the common pairing of any two representations (2.4) of $m$ is the same, since a vanishing subsum of $x_{0}+x_{1}-x_{2}-x_{3}$ with positive rational numbers $x_{0}, x_{1}, x_{2}, x_{3}$ and $x_{0}+$ $x_{1}-x_{2}-x_{3}=0$ yields $x_{0}=x_{2}, x_{1}=x_{3}$ or $x_{0}=x_{3}, x_{1}=x_{2}$.

For each possible common pairing we shall check the possible splittings in representations of the form $2^{\alpha} 3^{\beta}+2^{\gamma}+3^{\delta}$.

Case (b1). $m=2^{a} 3^{b}+c$ with $a, b \in \mathbf{Z} \backslash\{0\}, c \in\{5,11,17,35,259\}$. Here $c$ has to be split as $2^{\gamma}+3^{\delta}$. By Lemma 5 there are at most two distinct representations $2^{\alpha} 3^{\beta}+2^{\gamma}+3^{\delta}$ of $m$. 
Case (a2). $m=2^{a}+3^{b}$ with $a, b \in \mathbf{Z}$. If $a=0$, then $b$ is large and a splitting $3^{b}=2^{\alpha} 3^{\beta}+2^{\gamma}$ is impossible by Lemma 2 . If $b=0$ then $a$ is large and a splitting $2^{a}=2^{\alpha} 3^{\beta}+3^{\delta}$ is impossible, also by Lemma 2. Therefore the splitting is either $2^{a}=2^{\alpha} 3^{\beta}+2^{\gamma}$ or $3^{b}=2^{\alpha^{\prime}} 3^{\beta^{\prime}}+3^{\delta^{\prime}}$. By Lemma 2, the first possibility can be achieved in at most two ways, $2^{a-1} 3^{0}+2^{a-1}$ and $2^{a-2} 3^{1}+2^{a-2}$, and the second also in at most two ways, $2^{1} 3^{b-1}+3^{b-1}$ and $2^{3} 3^{b-2}+3^{b-2}$. They are given in the statement of Theorem 4 .

Case (a1). We may assume $m=2^{a} 3^{b}+c$ with $a \in \mathbf{N}, b \in\{-1,1,2\}$, $c \in\{3,9\}$. (The case $b=0$ has been treated in the preceding case.) The only possible splitting of $c$ is $2^{1}+3^{0}$ if $c=3$ and $2^{3}+3^{0}$ if $c=9$. By Lemma 2, there is only one splitting, $2^{a} 3^{-1}=2^{a-2} 3^{-1}+2^{a-2}$ if $b=-1$, only one splitting, $2^{a} 3^{1}=2^{a+1} 3^{0}+2^{a}$ if $b=1$, and only one splitting, $2^{a} 3^{2}=2^{a+3} 3^{0}+2^{a}$ if $b=2$. Here we use that the splitting should be of the form $2^{\alpha} 3^{\beta}+2^{\gamma}$. Thus there are at most two distinct representations $2^{\alpha} 3^{\beta}+2^{\gamma}+3^{\delta}$ of $m$.

Case (a4). We may assume $m=2^{a} 3^{b}+c$ with $a \in\{-3,-1,1,2\}$, $b \in \mathbf{N}, c \in\{2,4\}$. The only possible splitting of $c$ of the form $2^{\gamma}+3^{\delta}$ is $2^{0}+3^{0}$ if $c=2$ and $2^{0}+3^{1}$ if $c=4$. Otherwise $2^{a} 3^{b}$ should be split as $2^{\alpha} 3^{\beta}+3^{\delta}$. By Lemma 2 there is only one splitting for each value of a. Thus there are at most two distinct representations $2^{\alpha} 3^{\beta}+2^{\gamma}+3^{\delta}$ of $m$.

Case (b2). We may assume $m=2^{a}+3^{b} c$ with $a, b \in \mathbf{Z}, c \in$ $\{13 / 4,5,7,11,13,17,19,25,35,43,73,97,145,259\}$. Obviously $3^{b} c$ should be split. If $a \leq 0$, then $b$ is large and a splitting of $3^{b} c$ as $2^{\alpha} 3^{\beta}+2^{\gamma}$ is impossible by Lemma 4 , for $m>M_{4}$ say. Thus $3^{b} c=2^{\alpha} 3^{\beta}+3^{\delta}$. Put $B=\min (b, \beta, \delta)$. If $b=\beta=B$, then we have $c=2^{\alpha}+3^{\delta-B}$ which has at most two solutions, since the values $2^{x}+3^{y}$ of the solutions of Lemma 5 are distinct. If $b=\delta=B$, then $c=2^{\alpha} 3^{\beta-B}+1$, which gives at most one solution. If $\beta=\delta=B$, then $3^{b-B} c=2^{\alpha}+1$. Suppose $3^{b} c=2^{\alpha_{1}} 3^{B_{1}}+3^{B_{1}}=2^{\alpha_{2}} 3^{B_{2}}+3^{B_{2}}$ with $B_{1}<B_{2}$. Then $2^{\alpha_{1}}+1=2^{\alpha_{2}} 3^{B_{2}-B_{1}}+3^{B_{2}-B_{1}}$. By Theorem 3, there are at most two possible splittings for any $c$, since the 3-free parts of $2^{2}+1,2^{4}+1,2^{5}+1,2^{7}+1,2^{9}+1$ are distinct. Then there are at most two distinct representations of $m$.

Case (b3). We may assume $m=2^{a} c+3^{b}$ with $a, b \in \mathbf{Z}, c \in$ $\{7 / 3,5,7,73 / 9,11,13,17,19,25,35,41,73,97,145,259\}$. By a similar 
reasoning as in Case (b2) but applying Theorem 2 instead of Theorem 3, we reach the conclusion that there are at most three distinct representations of $m$.

3. The number of representations of the form $\sum_{i=1}^{n} p_{1}^{k_{i 1}} \cdots p_{t}^{k_{t 1}}$. Let $T=\left\{p_{1}, \ldots, p_{t}\right\}$ be a finite set of prime numbers. Let $S$ be the set of integers of the form $p_{1}^{k_{1}} \ldots p_{t}^{k_{t}}$ with $k_{1}, \ldots, k_{t} \in \mathbf{Z}$.

Theorem 5. There exists a number $C$ depending only on $n$ and $T$ such that every rational number has at most $C$ distinct representations as sum of $n$ elements from $S$.

More generally, we consider an algebraic number field $K$ of finite degree. Let $M_{K}$ be the set of places on $K$ (i.e. equivalence classes of multiplicative valuations on $K$ ). Let $S_{K}$ be a finite subset of $M_{K}$ containing all infinite places. Write

$$
S=\left\{\left.\alpha \in K|| \alpha\right|_{v}=1 \text { for all } v \notin S_{K}\right\} .
$$

Clearly, $S$ is a multiplicative subgroup of $K^{*}(=K \backslash\{0\})$. Let $\mathbf{P}^{n}(S)$ be the set of projective points $\left(x_{0}: x_{1}: \cdots: x_{n}\right)$ where the homogeneous coordinates are in $S$ and determined up to a multiplicative factor in $S$. We shall apply the following generalization of Lemma 4 .

LEMMA 6. There are only finitely many projective points $\left(x_{0}: x_{1}\right.$ : $\left.\cdots: x_{n}\right) \in \mathbf{P}^{n}(S)$ satisfying

$$
x_{0}+x_{1}+\cdots+x_{n}=0
$$

but

$$
\begin{gathered}
x_{i_{1}}+\cdots+x_{i_{k}} \neq 0 \text { for each proper non-empty subset } \\
\left.\qquad i_{1}, \ldots, i_{k}\right\} \text { of }\{0,1, \ldots, n\} .
\end{gathered}
$$

Proof. See van der Poorten and Schlickewei [7] and Evertse [5].

We shall prove the following generalization of Theorem 5 .

THEOREM 6. For every finite subset $W$ of $K^{*}$ there exists a number $C_{0}$ depending only on $n, S$ and $W$ such that every algebraic number in $K^{*}$ has at most $C_{0}$ distinct representations of the form

$$
w_{1} s_{1}+\cdots+w_{n} s_{n} \text { with } w_{1}, \ldots, w_{n} \in W, s_{1}, \ldots, s_{n} \in S
$$

without vanishing subsums. 
REMARKS. (1) The restriction 'without vanishing subsums' is necessary in view of the following example. Take $K=\mathbf{Q}$ and let $S$ correspond to the ordinary absolute value and the prime numbers 2 and 3. The number $\alpha=1$ has infinitely many distinct representations

$$
3.2^{k}-2^{k+1}-2^{k}+1 \quad(k \in \mathbf{Z}) \text {. }
$$

(2) Obviously, Theorem 5 is a special case of the above theorem with $K=\mathbf{Q}, W=\{1\}$.

Proof of Theorem 6. We apply induction on $n$. Clearly, the assertion is true for $n=1$. Now suppose that the assertion holds for all positive integers less than $n$. We shall show that it also holds for $n$. In the proof, $C_{0}(n, S, W), C_{1}(n, S, W), C_{2}(n, S, W), \ldots$ denote positive numbers which depend only on the indicated parameters. For any finite subset $W$ of $K^{*}$ there is a finite subset $S_{K}(W)$ of $M_{K}$ such that $W \subset S(W)$, where

$$
S(W)=\left\{\left.\alpha \in K|| \alpha\right|_{v}=1 \text { for all } v \notin S_{K}(W)\right\} .
$$

Thus Lemma 6 can be applied to the multiplicative group $S(W) S=$ $\left\{s_{1} s_{2} \mid s_{1} \in S(W), s_{2} \in S\right\}$.

Let $\alpha$ be a number in $K^{*}$ having two distinct representations of the form (3.1) each without vanishing subsums,

$$
\alpha=w_{1} s_{1}+\cdots+w_{n} s_{n}=w_{1}^{\prime} s_{1}^{\prime}+\cdots+w_{n}^{\prime} s_{n}^{\prime} .
$$

Then

$$
w_{1} s_{1}+\cdots+w_{n} s_{n}-w_{1}^{\prime} s_{1}^{\prime}-\cdots-w_{n}^{\prime} s_{n}^{\prime}=0 .
$$

Suppose that there exist $r$ disjoint subsets $I_{1}, \ldots, I_{r}$ in $I=\{1, \ldots, n\}$ and corresponding disjoint subsets $J_{1}, \ldots, J_{r}$ such that

$$
\begin{gathered}
\bigcup_{l=1}^{r} I_{l}=\bigcup_{l=1}^{r} J_{l}=I, \\
\sum_{i \in I_{l}} w_{i} s_{i}-\sum_{j \in I_{l}} w_{j}^{\prime} s_{j}^{\prime}=0 \quad \text { for } l=1, \ldots, r,
\end{gathered}
$$

but the left hand side of (3.3) has no vanishing subsums. If $r=n$ then $g_{l}+h_{l}=2$ for all $l=1, \ldots, r$, where $g_{l}, h_{l}$ denote the cardinal numbers of $I_{l}$ and $J_{l}$, respectively. Therefore the two representations are not distinct, have vanishing subsums or $\alpha$ is zero. This is a contradiction 
with the hypothesis. Thus we have $1 \leq r \leq n-1$. Now apply Lemma 6 to each equation in (3.3). Hence there are only finitely many projective points in $\mathbf{P}^{g_{l}+h_{l}-1}(S(W) S)$ :

$$
\left(w_{i_{11}} s_{i_{l 1}}: \cdots: w_{i_{l_{l}}} s_{i_{l_{g_{l}}}}: w_{j_{l_{1}}}^{\prime} s_{j_{l_{1}}}^{\prime}: \cdots: w_{j_{l_{l}}}^{\prime} s_{j_{l h_{l}}}^{\prime}\right)
$$

for $l=1, \ldots, r$ such that the corresponding equations (3.3) hold. Hence we have only finitely many points in $\{1\} \times(S(W) S)^{g_{l}-1}$ :

$$
\left(1,\left(w_{i_{11}} s_{i_{11}}\right)^{-1} w_{i_{l 2}} s_{i_{l 2}}, \ldots,\left(w_{i_{l 1}} s_{i_{11}}\right)^{-1} w_{i_{l_{l}}} s_{i_{l_{l}}}\right)
$$

for $l=1, \ldots, r$ such that the corresponding equations (3.3) hold. Put

$$
u_{l}=1+\sum_{k=2}^{g_{l}}\left(w_{i_{l 1}} s_{i_{11}}\right)^{-1} w_{i_{l k}} s_{i_{l k}} \quad \text { for } l=1, \ldots, r .
$$

Since the representations have no vanishing subsums, $u_{1}, \ldots, u_{r}$ are all non-zero. Clearly they belong to a finite set $W^{\prime}$ of $K^{*}$ which depends only on $n, S$ and $W$. (Since the number of splittings depends only on $n$, we can make $W^{\prime}$ independent of $r$ and the particular splitting by taking the union of elements $u_{1}, \ldots, u_{r}$ over all splittings.) Note that

$$
\alpha=\sum_{l=1}^{r}\left(w_{i_{l 1}} s_{i_{l 1}}\right) u_{l}=\sum_{l=1}^{r}\left(w_{i_{l 1}} u_{l}\right) s_{i_{l 1}},
$$

where $1 \leq r \leq n-1$ and $w_{i_{11}} u_{l}(1 \leq l \leq r)$ belong to a finite set $W^{\prime \prime}$ of $K^{*}$ which depends only on $n, S$ and $W$. Note that the sums in (3.4) have no vanishing subsums. According to the induction hypothesis we conclude that there exist numbers $C_{0}\left(r, S, W^{\prime \prime}\right)$ such that $\alpha$ has at most

$$
C_{1}(n, S, W):=\sum_{r=1}^{n-1} C_{0}\left(r, S, W^{\prime \prime}\right)
$$

distinct representations of the form (3.4) with

$$
w_{i_{l}} u_{l} \in W^{\prime \prime}, s_{i_{1}} \in S \text { for } l=1, \ldots, r .
$$

Because the number of ordered splittings of $I$ in subsets $I_{1}, \ldots, I_{r}$ as well as in $J_{1}, \ldots, J_{r}$ is bounded by a number $C_{2}(n)$, we have that $\alpha$ has at most

$$
C_{3}(n, S, W)=C_{2}^{2}(n) C_{1}(n, S, W)
$$

pairs of distinct representations of the form (3.1). 


\section{REFERENCES}

[1] L. J. Alex, Diophantine equations related to finite groups, Comm. Algebra, 4 (1976), 77-100.

[2] J. L. Brenner and L. L. Foster, Exponential diophantine equations, Pacific J. Math., 101 (1982), 263-301.

[3] W. J. Ellison, Recipes for solving diophantine problems by Baker's method, Sém. Théorie des Nombres, Univ. Bordeaux I, 1970-71, Lab. Th. Nombr. C.N.R.S., Exp. 11,10 pp.

[4] P. Erdös and R. L. Graham, Old and new problems and results in combinatorial number theory, Monographie No. 28 de L'Enseignement Mathématique, Kundig, Genève, 1980.

[5] J.-H. Evertse, On sums of S-units and linear recurrences, Compositio Math., 53 (1984), 225-244.

[6] J.-H. Evertse, K. Györy, C. L. Stewart and R. Tijdeman, S-unit equations and their applications, in: New Advances in Transcendence Theory, ed. by A. Baker, Cambridge Univ. Press, 1988.

[7] A. J. van der Poorten and H. P. Schlickewei, The growth conditions for recurrence sequences, Macquarie Univ. Math. Rep. 82-0041, North Ryde Australia, 1982.

[8] P. Vojta, Integral points on varieties, Thesis, Harvard, 1983.

[9] R. J. Stroeker and R. Tijdeman, Diophantine equations, Computational Methods in Number Theory, M. C. Tract 155, Centr. Math. Comp. Sci., Amsterdam, (1982), 321-369.

[10] B. M. M. de Weger, Solving exponential diophantine equations using lattice basis reduction algorithms, J. Number Theory, 26 (1987), 325-367.

Received October 24, 1986 and in revised form March 18, 1987.

Mathematical Institute

R. U. LEIDEN

2333 CA LEIDEN

THE NETHERLANDS

AND

INSTITUTE OF MATHEMATICS

Academia Sinica

Beijing, China 



\section{PACIFIC JOURNAL OF MATHEMATICS \\ EDITORS}

V. S. VARADARAJAN

(Managing Editor)

University of California

Los Angeles, CA 90024

Herbert Clemens

University of Utah

Salt Lake City, UT 84112

R. FINN

Stanford University

Stanford, CA 94305
HERMANN FLASCHKA

University of Arizona

Tucson, AZ 85721

RAMESH A. GANGOLLI

University of Washington Seattle, WA 98195

VAUGHAN F. R. JONES

University of California

Berkeley, CA 94720
ROBION KIRBY

University of California

Berkeley, CA 94720

C. C. MOORE

University of California

Berkeley, CA 94720

HAROLD STARK

University of California, San Diego

La Jolla, CA 92093

\section{ASSOCIATE EDITORS}
R. ARENS
E. F. BECKENBACH
B. H. NEUMANN
F. WOLF
K. YOSHIDA
(1906-1982)

\section{SUPPORTING INSTITUTIONS}

UNIVERSITY OF ARIZONA

UNIVERSITY OF BRITISH COLUMBIA

CALIFORNIA INSTITUTE OF TECHNOLOGY

UNIVERSITY OF CALIFORNIA

MONTANA STATE UNIVERSITY

UNIVERSITY OF NEVADA, RENO

NEW MEXICO STATE UNIVERSITY

OREGON STATE UNIVERSITY
UNIVERSITY OF OREGON UNIVERSITY OF SOUTHERN CALIFORNIA

STANFORD UNIVERSITY

UNIVERSITY OF HAWAII

UNIVERSITY OF TOKYO

UNIVERSITY OF UTAH

WASHINGTON STATE UNIVERSITY

UNIVERSITY OF WASHINGTON 


\section{Pacific Journal of Mathematics \\ Vol. 132, No. $1 \quad$ January, 1988}

Scott W. Brown, Full analytic subspaces for contractions with rich spectrum ...1

Robert Main Burton, Jr. and Tae-Sung Kim, An invariance principle for

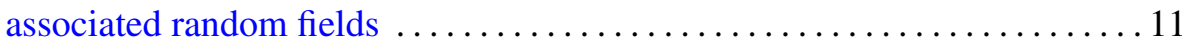

Ana M. Viola-Prioli and Jorge Viola-Prioli, Rings whose kernel functors

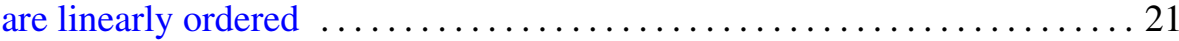

David E. Handelman, Representing polynomials by positive linear

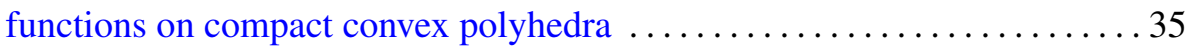

Patrick Keef, On the Tor functor and some classes of abelian groups . .....66

Dennis R. Malm, Simplicity of partial and Schmidt differential operator

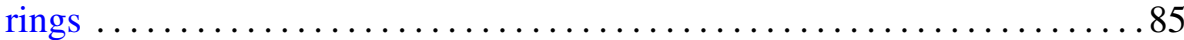

José M. Montesinos and Carmen Safont, On the Birman invariants of

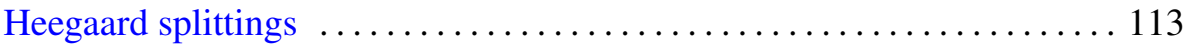

N. P. Mukherjee and Prabir Bhattacharya, The normal index of a finite group

Mario Petrich and Norman R. Reilly, Semigroups generated by certain operators on varieties of completely regular semigroups

Robert Tijdeman and Lian Xiang Wang, Sums of products of powers of given prime numbers

Joel Larry Weiner, First integrals for a direction field on a simply connected plane domain 